\title{
Contribution of bronchoscopic lavage to the diagnosis of smear negative pulmonary tuberculosis
}

\author{
Mustafa Engin Şahin $\odot$, Sertan Bulut $\odot$ \\ Department of Chest Diseases, Atatürk Chest Diseases and Chest Surgery Training and Research Hospital, Ankara, Turkey
}

\begin{abstract}
Objectives: Sputum acid-fast bacilli (AFB) and Xpert examinations are the most commonly used methods in early diagnosis for pulmonary tuberculosis. It becomes difficult for the clinician to initiate treatment in patients with negative sputum smear and Xpert examinations at the stage of diagnosis. Waiting for culture results for diagnosis causes delay in treatment and increases the risk of disease transmission. We aimed to investigate the contribution of bronchoscopic lavage (BL) obtained with flexible fiberoptic bronchoscopy (FOB) to the diagnosis of tuberculosis.

Methods: The BL results of 36 patients hospitalized in the tuberculosis clinic who were clinically suspected of tuberculosis, but whose sputum AFB and Xpert results were negative, and whose bronchoscopic lavages were taken by FOB, were retrospectively evaluated.

Results: BL Xpert examination was the method that made the most contribution to the diagnosis with a rate of $27.7 \%$. BL smear AFB results did not contribute to the diagnosis statistically. Sputum and BL tuberculosis culture results were found to be close to each other. In total, the diagnosis of $50 \%$ of the patients was confirmed. Conclusions: Performing Xpert examinations on bronchoscopic materials of patients with negative sputum examinations significantly increase the rate of early diagnosis.

Keywords: Sputum, smear negative, pulmonary tuberculosis, acid-fast bacilli, bronchoalveolar lavage, MGIT Culture, Xpert
\end{abstract}

T uberculosis (TB) is one of the top 10 causes of death worldwide, and nearly a quarter of the world's population is infected with Mycobacterium tuberculosis [1]. Although mycobacterium culture is the gold standard in the diagnosis of pulmonary tuberculosis, the growth of M. tuberculosis in cultures occurs after 6-8 weeks. Therefore, sputum acid-fast bacilli (AFB) and Xpert examinations are the most commonly used methods in early diagnosis. Sputum smear is important for early diagnosis, but in order to observe the bacillus in the smear, there should be 5000 bacilli per $\mathrm{ml}[2]$. Approximately $50-60 \%$ of patients with pulmonary tuberculosis can be diagnosed with sputum smear examinations [3]. Xpert test is more sensitive than microscopy [4]. However, even if there is a bacillus in the sputum, a small number of bacilli may not be detected. It becomes difficult for the clinician to initiate treatment in patients with negative sputum smear and Xpert examinations at the stage of diagnosis. Waiting for culture results for diagnosis both causes delay in treatment and increases the risk of disease transmission. Flexible fiberoptic bronchoscope (FOB) can be used to perform mycobacterial examinations in these patients $[5,6]$. In addition, broncho-

How to cite this article: Şahin ME, Bulut S. Contribution of bronchoscopic lavage to the diagnosis of smear negative pulmonary tuberculosis. Eur Res J 2022;8(6):845-850. DOI: 10.18621/eurj.870909

Address for correspondence: Mustafa Engin Şahin, MD., Atatürk Chest Diseases and Chest Surgery Training and Research Hospital, Department of Chest Diseases, Ankara,Turkey. E-mail: drenginsahin@protonmail.com, Tel (Mobil): +90 5064074328 
scopic lavage (BL), broncho-alveolar lavage (BAL), and bronchial brushing methods can be applied for early diagnosis. Considering the efficacy/complication rate, trans-bronchial needle aspiration (TBNA) or trans-thoracic needle aspiration biopsy (TNAB) has been used for a long time because of its contribution to both early diagnosis and differential diagnosis [710]. These methods are important both in detecting tuberculosis and in differential diagnosis. Averagely $30-40 \%$ untreated smear negative patient will develop active TB within first two year period [11].

\section{METHODS}

\section{Patients}

In the institution where we work, a committee called the Medical Specialty Education Board is convened and it checks the appropriateness of the planned studies. So we applied to this board and got the approval of our study with the decision (date-number Jan 25, 2020-659). Thirty-six patients with a clinical and radiological suspicion of pulmonary tuberculosis, who received inpatient treatment at our Ankara Ataturk Chest Diseases and Thoracic Surgery Training and Research Hospital 2-TB clinic between 2018-2019 years, and whose smear AFBs and sputum Xperts found negative in 3 sputum samples was included. Sputum induction was applied to all patients who could not produce sputum or had negative sputum smears. The additional contribution of BL application to sputum examinations was investigated. Therefore, patients who could not produce sputum in any way were excluded from the study. Less involvement in the upper right zone was evaluated as limited involvement on chest radiography. Those with more involvement were defined as diffuse involvement. Those with and without cavities in the radiography were recorded. Smear AFB and Xpert positivity of BL were accepted as early diagnosis.

We included 36 patients who had negative sputum AFB and Xpert's but had a pre-diagnosis of pulmonary tuberculosis to our study and we aimed to evaluate the contribution of smear, culture and Xpert results of their FOB lavages' to the diagnosis.

\section{Bronchoscopic Lavage Technique}

Written informed consent was obtained from all patients before the bronchoscopic procedure. Premedication was performed with diazepam before FOB. Also local anesthesia was applied to the upper respiratory tract with $10 \%$ lidocaine. After examining the tracheobronchial tree with Olympus 1T260 type FOB, BL was performed with 15-20 cc saline solution from the lobe bronchus, where lesions were detected by radiological methods before the procedure. During the procedure, $2 \%$ lidocaine was applied as needed for local anesthesia. Lidocaine was not applied during lavage.

\section{Microbiological Testing}

The lavage smears were stained with ZiehlNeelsen (Z-N) to search for acid-fast bacilli. BL samples were seeded on solid (Lowenstein-Jensen) and liquid (MGIT 960) media for tuberculosis culture.

\section{Xpert MTB/RIF Test}

This test is a nucleic acid amplification (NAA) test. Disposable cartridge is used. It is based on heminested real-time PCR that amplifies the rpoB gene target. Sample is taken from the patient with suspected TB. The material is mixed with the reagent provided by the test and a cartridge containing this mixture is placed in the Xpert machine. From this point on, all transactions are fully automated. The Xpert MTB/RIF test detects Mycobacterium Tuberculosis Complex (MTBC) and rifampine (RIF) resistance in less than 2 hours. It has an excellent accuracy when made in sputum and has been approved by the World Health Organization (WHO) $[12,13]$. The general sensitivity and specificity of the Xpert Ultra test we used were reported as $87.5 \%$ and $98.7 \%$ respectively. This sensitivity was found to be $78.9 \%$ in smear negative samples. The limit of detection (LOD) is reported as 15.6 $\mathrm{CFU} / \mathrm{ml}$ for sputum [14].

Cepheid Xpert MTB / RIF Ultra, GXMTB / RIFULTRA-10 devices and cartridges were used for Xpert evaluation. After the material was treated with $\mathrm{NaOH}$, it was mixed with the reagent provided with the test and loaded into the system with a disposable cartridge and the samples were evaluated by automatic reading by the system.

\section{Statistical Analysis}

Categorical independent data were analyzed using the chi-square test. Categorically dependent data were 
analyzed using the McNemar test. Normal distribution of numeric variables was evaluated with Shapiro-Wilk test, Skewness and Kurtosis. Normally-distributed numeric variables were expressed as mean and standard variation while non-normally distributed variables were expressed as median. The correlation between two numeric variables with normal distribution and a linear correlation was analyzed using Pearson's test. The correlation between variables that did not show normal distribution was evaluated with Spearman's test. All analyses were performed using SPSS 22.0 statistical software (SPSS Inc, Chicago, IL, USA). A $p<$ 0.05 was considered statistically significant.

\section{RESULTS}

Of the patients included in the study, $17(47.2 \%)$ were female and $19(52.7 \%)$ were male. The mean age of the female patients was 42.2 and the mean age of the male patients was 47.9. The youngest patient was 18 and the oldest patient was 83 years old. The most common symptom was cough $(88.9 \%)$, followed by weight loss (69.4\%) and fever (55.6\%) (Table 1).

Comorbid diseases are chronic obstructive pulmonary disease (1 patient), asthma bronchiale (1 patient), cervix $\mathrm{Ca}$. (1 patient) and non-hodgkin lymphoma (1 patient). Two patients were diagnosed

\section{Table 1. Demography and symptoms}

\begin{tabular}{cc}
\hline & Data \\
\hline Gender, $\mathrm{n}(\%)$ & $19(52.7)$ \\
\hline Male & $17(47.2)$ \\
\hline Female & \\
Age (years) (mean \pm SD) & $47.94 \pm 22.27$ \\
\hline Male & $42.11 \pm 19.64$ \\
\hline Female & \\
Symptoms, $(\%)$ & $32(88.9)$ \\
\hline Cough & $25(69.4)$ \\
\hline Weight loss & $20(55.6)$ \\
\hline Fever & $17(47.2)$ \\
\hline Weakness & $7(19.4)$ \\
\hline Hemoptysis & $4(11.1)$ \\
\hline Dyspnea & $2(5.6)$ \\
\hline Chest pain & \\
\hline
\end{tabular}

with diabetes mellitus and were using oral anti-diabetic agents. None of the patients were HIV positive but close contact was detected in the histories of six patients. There was a cavity in the chest radiograph of 21 patients. There was no correlation between the presence of cavity and BLAFB smear/Xpert or culture positivity ( $p=0.768$ and $p=0.571$, respectively). Radiologically, seven patients had less involvement than right upper zone. Wider involvement was detected in 29 patients (Table 2). The relationship between the bacteriological positivity rates of the patients and the CRP, sedimentation rate, PPD values, the presence of cavity in the chest radiographs and the amount of involved zones were evaluated. There was no statistically significant relationship between these parameters $(p=0.484, p=0.733, p=0.059$ and $p=/ 0.079$ respectively).

Sputum tuberculosis culture result was positive in $12(33.3 \%)$ patients. On the other hand, lavage tuberculosis culture was positive in $9(25 \%)$ patients. There was no statistically significant difference between lavage and smear culture positivity $(p>0.05)$. In one patient, MBT and non-tuberculous mycobacteria (NTM) were found together in mycobacterial cultures. In $3(5.5 \%)$ patients, lavage smear was positive for AFB. This rate was not statistically significant $(p=$ 0.25 ). Two of these 3 patients had positive lavage Xpert results. In the third patient, the sputum culture was positive, so lavage AFB contributed to early diagnosis in only 1 patient. In $10(27.7 \%)$ patients, lavage Xpert was positive. This rate was statistically significant $(p=0.002)$.

The examinations of $13(36.1 \%)$ patients were positive and $11(30.5 \%)$ patients were diagnosed early with the applied BL. Most of the patients who can be diagnosed early are diagnosed with Xpert positivity. AFB positivity in bronchoscopic lavage was detected in only $3(8.3 \%)$ cases. With lavage Xpert and AFB positivity, this rate was $30.5 \%$ with 11 patients (Table $3)$. In total, $18(50 \%)$ patients were diagnosed with sputum culture and lavage examinations. Clinical improvement and radiological regression were detected in all patients during their 6-month follow-up.

\section{DISCUSSION}

In this study, we aimed to evaluate the contribution of 
Table 2. Bacteriological positivity according to chest radiography findings

\begin{tabular}{|c|c|c|c|c|c|c|}
\hline$(n=36)$ & 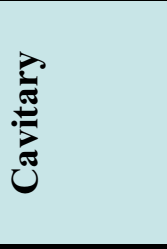 & ל্ট & 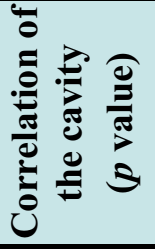 & 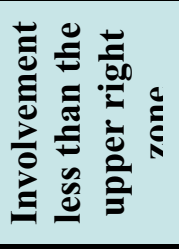 & 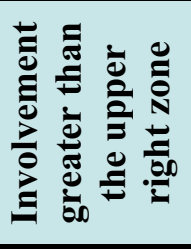 & 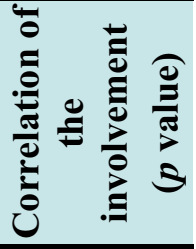 \\
\hline Chest radiography, n (\%) & $21(58.3)$ & $15(41.6)$ & & $7(19.4)$ & $29(80.6)$ & \\
\hline BL AFB smear or Xpert + & 6 & 4 & 0.768 & 1 & 9 & 0.311 \\
\hline BL Culture + & 5 & 4 & 0.571 & 0 & 9 & 0.094 \\
\hline
\end{tabular}

$\mathrm{AFB}=$ Acid fast bacilli, $\mathrm{BL}=$ Bronchoscopic lavage

bronchial lavage results to the diagnosis of pulmonary tuberculosis in patients with negative AFB smear in sputum.

In our study, lavage smear AFB was found to be positive in $3(8.3 \%)$ patients. Two of these three patients had positive lavage Xpert results and one had positive sputum culture results. Previous studies show that BL smear AFB exams can range from $4 \%$ to $60 \%$ $[6,15,16]$. In the study of Yuksekol et al. [17], one of the studies conducted in our country, AFB direct examination of bronchial lavage was found to be positive in $23.2 \%$ of cases. While this rate was $19 \%$ in Alp et al.'s [8] study, Balbay et al. [7] Found that BL smear positivity rate was $29 \%$ which was higher than ours. However, in the study of Intepe et al. [18], bronchoalveolar lavages of 83 patients were examined and no smear positivity was found in any of the patients. Studies show that the rate of AFB smear positivity in $\mathrm{BL}$ or BAL can vary in a wide range.

Although it has been reported that bronchoscopic examinations may contribute more to the diagnosis than sputum examinations, the positivity of induced sputum smear is considerably higher. In the comparative study published in 2017 by Luhadia et al. [19], a microbiological comparison of induced sputum and bronchial aspirate was performed on 100 patients (60/40, respectively) with negative sputum smear AFB results, and the diagnostic value of bronchial aspirate was found to be significantly higher. It has been suggested that FOB examinations can be preferred according to the cost-complication/efficiency ratio. In the study of Gopathi et al. [20], the positivity of AFB smear in BL is slightly higher than in induced sputum. The results of sputum induction and bronchoscopy were similar in the meta-analysis performed by Luo et al. [21].

We think that our AFB smear results can be compared with the results of Luhadia, Gopathi and Lua's studies, as sputum induction is applied to patients who cannot produce sputum or who are negative for sputum AFB smears.

In our study, smear AFB results were positive only in 3 patients, but when this result is evaluated together with BL AFB, smear and Xpert results, the positivity rate increases from $8.33 \%$ to $30.5 \%$. This ratio is also the rate of patients who were diagnosed early. Early diagnosis was obtained mostly $(27.7 \%)$ by Xpert positivity.

The Xpert MTB / RIF tests, which started to become widespread in the 2000s and are now being used in many centers in our country, can detect a higher rate of positivity in materials taken with FOB than in AFB smear examinations [22-25]. There are many publications that support this. In the study of Lee et al. [26]

Table 3. Bacteriological results

\begin{tabular}{lccc}
\hline & $\begin{array}{c}\text { Smear AFB } \\
\text { n (\%) }\end{array}$ & $\begin{array}{c}\text { Xpert }+ \\
\text { n (\%) }\end{array}$ & $\begin{array}{c}\text { Solid and liquid culture + } \\
\text { n (\%) }\end{array}$ \\
\hline Sputum $(\mathrm{n}=36)$ & $0(0)$ & $0(0)$ & $12(33.3)$ \\
BL $(\mathrm{n}=36)$ & $3(8.3)$ & $10(27.7)$ & $9(25)$ \\
\hline
\end{tabular}

$\mathrm{AFB}=$ Acid fast bacilli, $\mathrm{BL}=$ Bronchoscopic lavage 
published in 2013, 50 of 132 patients were diagnosed with tuberculosis with BL + BAL, and the sensitivity of AFB smear and Xpert tests in this study was found to be $13.2-81.6 \%$, respectively. In the study of Khalil and Butt [24] these rates were found to be $36 \%$ and $87 \%$, respectively. In the study published by Le Palud et al. [23] in 2014 and evaluated 162 patients retrospectively, BL or BAL were used as bronchoscopic material. In this study in which 30 tuberculosis patients were identified, $16 \%$ of the patients were AFB smear positive, while $80 \%$ were found to be Xpert positive [23]. However, the sensitivity of the Xpert test in smear-negative samples is reduced [14].

The patients included in almost all of the other studies were those who sesputum smears were negative or who were unable to produce sputum. However, patients who could not produce sputumwere not included in our study. We think this is an important reason why our BL smear results were less positive than previous studies. Although the sputum examinations of all patients were examined together with the AFB smear and the Xpert, the negative sputum examinations suggest that the number of bacilli in the respiratory secretions of the patients included in the study may be low. This is also observed in our FOB-BL results.

The inhibitory effect of lidocaine, which is used as a local anesthetic in bronchoscopy, to the growth of MTB in cultures, has been known for a long time [2729]. The possibility that the patients may have a low number of bacilli in respiratory secretions or that the patients have not been able to produce high-quality sputum do not completely eliminate the possibility of lidocaine inhibition. In the study of Schmidt et al. [30], it is stated that lidocaine at concentrations of $1 \%$ and above inhibits MBT growth. In our study, we tried to minimize the local anesthetic exposure of the bacilli during the bronchoscopic procedure. In addition, positive results of sputum and BL cultures decrease this possibility. The results of our study exclude the negative effect of local anesthesia, with a lower rate of smear-AFB positivity in lavage, and a higher rate of culture positivity. We consider the inhibition of MTB growth due to lidocaine as a poor possibility.

In our study, BL AFB smear examinations did not significantly contribute to early diagnosis. However, the Xpert results made a statistically and clinically sig- nificant positive contribution. Although the disadvantage of Xpert examinations is that they can not detect non-tuberculosis mycobacteria, it's advantage is the detection of rifampicin resistance and rapid results less than two hours.

\section{CONCLUSION}

Bronchoscopic lavage Xpert examination contributes significantly more to the diagnosis than sputum Xpert examination. In centers where Xpert MTB/RIF can be examined, bronchial lavage statistically increases the rate of early diagnosis. It is also important to learn about RIF resistance at an early stage, in terms of the continuation and direction of treatment.

\section{Authors' Contribution}

Study Conception: MEŞ, SB; Study Design: MEŞ, SB; Supervision: MEŞ, SB; Funding: N/A; Materials: MEŞ, SB; Data Collection and/or Processing: MEŞ, SB; Statistical Analysis and/or Data Interpretation: MEŞ, SB; Literature Review: MEŞ, SB; Manuscript Preparation: MEŞ, SB and Critical Review: MEŞ, SB.

\section{Conflict of interest}

The authors disclosed no conflict of interest during the preparation or publication of this manuscript.

\section{Financing}

The authors disclosed that they did not receive any grant during conduction or writing of this study.

\section{REFERENCES}

1. Global Tuberculosis Report 2019. https://www.who.int/tb/global-report-2019. Accessed 27 Jun 2020.

2. Toman K. How many bacilli are present in a sputum specimen found positive by smear microscopy? In: Frieden T. editors. Toman's Tuberculosis Case detection, treatment, and monitoring - questions and answers, Geneva: World Health Organization; 2004, p. 11.

3. Siddiqi K, Lambert M-L, Walley J. Clinical diagnosis of smear-negative pulmonary tuberculosis in low-income countries: the current evidence. Lancet Infect Dis 2003;3:288-96.

4. Barnard DA, Irusen EM, Bruwer JW, Plekker D, Whitelaw AC, Deetlefs JC, et al. The utility of Xpert MTB/RIF performed on 
bronchial washings obtained in patients with suspected pulmonary tuberculosis in a high prevalence setting. BMC Pulm Med 2015;15:103.

5. Bachh A, Gupta R, Haq I, Varudkar HG. Diagnosing sputum/smear-negative pulmonary tuberculosis: Does fibre-optic bronchoscopy play a significant role? Lung India 2010;27:58.

6. Kacar N, Bicer B, Tuksavul F, Guclu SZ. [Diagnostic value of bronchial lavage, bronchoalveolar lavage and postbronchoscopic sputum in smear negative pulmonary tuberculosis]. Turkiye Klinikleri Arch Lung 2004;5:200-2. [Article in Turkish]

7. Balbay OA, Calışır HC, Erturk A, Ogretensoy M. [The diagnostic value of bronchial lavage, bronchial biopsy and postbronchoscopic sputum in smear-negative pulmonary tuberculosis]. Tüberküloz ve Toraks Dergisi 2001;49:124-8. [Article in Turkish]

8. Alp AI, Karlikaya C, Hatipoglu ON, Caglar T, Otkun M. [Diagnostic value of fiberoptic bronchoscopy in sputum smear negative pulmonary tuberculosis]. Tüberküloz ve Toraks Dergisi 2002;50:278-87. [Article in Turkish]

9. Willcox PA, Benatar SR, Potgieter PD. Use of the flexible fibreoptic bronchoscope in diagnosis of sputum-negative pulmonary tuberculosis. Thorax 1982;37:598-601.

10. Charoenratanakul S, Dejsomritrutai W, Chaiprasert A. Diagnostic role of fiberoptic bronchoscopy in suspected smear negative pulmonary tuberculosis. Respir Med 1995;89:621-3.

11. Pathakumari B, Prabhavathi M, Anbarasu D, Paramanandhan P, Raja A. Dynamic IgG antibody response to immunodominant antigens of $M$. tuberculosis for active TB diagnosis in high endemic settings. Clin Chim Acta 2016;461: 25-33.

12. Helb D, Jones M, Story E, Boehme C, Wallace E, Ho K, et al. Rapid detection of mycobacterium tuberculosis and rifampin resistance by use of on-demand, near-patient technology. J Clin Microbiol 2010;48:229-37.

13. World Health Organization. Automated real-time nucleic acid amplification technology for rapid and simultaneous detection of tuberculosis and rifampicin resistance: Xpert MTB/RIF system : policy statement. 2011. http://www.ncbi.nlm.nih.gov/books/NBK304235/ Accessed 29 Sep 2020.

14. Chakravorty S, Simmons AM, Rowneki M, Parmar H, Cao Y, Ryan J, et al. The new Xpert MTB/RIF ultra: improving detection of mycobacterium tuberculosis and resistance to rifampin in an assay suitable for point-of-care testing. mBio 2017;8:e00812-17.

15. Chan HS, Sun AJM, Hoheisel GB. Bronchoscopic aspiration and bronchoalveolar lavage in the diagnosis of sputum smearnegative pulmonary tuberculosis. Lung 1990;168:215-20.

16. Sanjeevaiah S, Haranal MY, Buggi S. Role of flexible bronchoscopy in patients with sputum-negative pulmonary tuberculosis. Indian J Thorac Cardiovasc Surg 2018;34:365-9.

17. Yüksekol İ, Bal Ş, Özkan M, Balkan A, Bedirhan I, Tozkoparan E, et al. [The value of fiberoptic bronchoscopy in diagnosis of smear negative pulmonary tuberculosis]. Tüberküloz ve Toraks Dergisi 2003;51:405-9. [Article in Turkish]

18. Intepe YS, Kıyık M, Tigin HC, Durmaz A, Epozturk K, C1krıkcıoglu S. [Diagnostic value acid fast bacilli at BAL in patients who are accepted as clinically and radiologically active tuberculosis with negative sputum smear]. Bozok Med J 2014;4:31-6. [Article in Turkish]

19. Luhadia A, Kapur M, Luhadia SK, Sharma RK. Comparison of induced sputum and fibre-optic bronchoscopy (Fob) in the early diagnosis of sputum smear negative suspected cases of pulmonary tuberculosis under Rntcp settings-A study conducted in southern part of Rajasthan. J Pulm Respir Med 2017;7:1000140 20. Gopathi NR, Mandava V, Namballa UR, Makala S. A comparative study of induced sputum and bronchial washings in diagnosing sputum smear negative pulmonary tuberculosis. J Clin Diagn Res 2016;10:OC07-10.

21. Luo W, Lin Y, Li Z, Shi Y. Comparison of sputum induction and bronchoscopy in diagnosis of sputum smear-negative pulmonary tuberculosis: a systemic review and meta-analysis. BMC Pulm Med 2020;20:146.

22. Tamura A, Shimada M, Matsui Y, Kawashima M, Suzuki J, Ariga $\mathrm{H}$, et al. The value of fiberoptic bronchoscopy in culturepositive pulmonary tuberculosis patients whose pre-bronchoscopic sputum specimens were negative both for smear and PCR analyses. Inter Med 2010;49:95-102.

23. Le Palud P, Cattoir V, Malbruny B, Magnier R, Campbell K, Oulkhouir Y, et al. Retrospective observational study of diagnostic accuracy of the Xpert ${ }^{\circledR}$ MTB/RIF assay on fiberoptic bronchoscopy sampling for early diagnosis of smear-negative or sputum-scarce patients with suspected tuberculosis. BMC Pulm Med 2014;14:137.

24. Khalil KF, Butt T. Diagnostic yield of bronchoalveolar lavage gene Xpert in smear- negative and sputum-scarce pulmonary tuberculosis. J Coll Physicians Surg Pak 2015;25:115-8.

25. Min JW, Yoon HI, Park KU, Song JH, Lee CT, Lee CH. Realtime polymerase chain reaction in bronchial aspirate for rapid detection of sputum smear-negative tuberculosis. Int J Tuberc Lung Dis 2010;14:852-8.

26. Lee HY, Seong MW, Park SS, Hwang SS, Lee J, Park YS, et al. Diagnostic accuracy of Xpert ${ }^{\circledR}$ MTB/RIF on bronchoscopy specimens in patients with suspected pulmonary tuberculosis. Int J Tuberc Lung Dis 2013;17:917-21.

27. Bartlett JG, Alexander J, Mayhew J, Sullivan-Sigler N, Gorbach SL. Should fiberoptic bronchoscopy aspirates be cultured? Am Rev Respir Dis 1976;114:73-8.

28. Danek SJ, Bower JS. Diagnosis of pulmonary tuberculosis by flexible fiberoptic bronchoscopy. Am Rev Respir Dis 1979;119:677-9.

29. Kvale PA, Johnson MC, Wroblewski DA. Diagnosis of tuberculosis: routine cultures of bronchial washings are not indicated. Chest 1979;76:140-2.

30. Schmidt RM, Rosenkranz HS. Antimicrobial activity of aocal anesthetics: lidocaine and procaine. J Infect Dis 1970;121:597607. 